\title{
Pharmacokinetics of the Yougui pill in experimental autoimmune encephalomyelitis model rats and its pharmacological activity in vitro
}

This article was published in the following Dove Press journal: Drug Design, Development and Therapy

\author{
Haolong Liu ${ }^{1,2, *}$ \\ Feng Qiu',* \\ Xinwei Yang' \\ Haiyu Zhao ${ }^{3}$ \\ Baolin Bian ${ }^{3}$ \\ Lei Wang'
}

'School of Traditional Chinese Medicine, Beijing Key Lab of TCM Collateral Disease Theory Research, Capital Medical University, Beijing 100069, China; ${ }^{2}$ Beijing Institute For Drug Control, Beijing Key Laboratory of Analysis and Evaluation on Chinese Medicine, Beijing I00035, China; ${ }^{3}$ Institute of Chinese Materia Medica, China Academy of Chinese Medical Sciences, Beijing 100700, China

*These authors contributed equally to this work
Correspondence: Baolin Bian Institute of Chinese Materia Medica, China Academy of Chinese Medical Sciences, Beijing 100700, China

Email blbian@icmm.ac.cn

Lei Wang

School of Traditional Chinese Medicine, Beijing Key Lab of TCM Collateral

Disease Theory Research, Capital Medical

University, Beijing 100069, China

Tel +86 I0 839| 1626

Fax +86 10 8391 1627

Email tmwangl@ccmu.edu.cn
Purpose: To determine the pharmacokinetic properties and pharmacological activity of the Yougui pill (YGP), which is a well-known Chinese medicine formula.

Methods: An ultra-performance liquid chromatography coupled with triple-quadrupole tandem mass spectrometry via electrospray ionization interface (UPLC-ESI-MS/MS) method was developed and validated for the simultaneous determination of several components in rat plasma. The method was then successfully applied to the pharmacokinetics of six bioactive components in experimental autoimmune encephalomyelitis (EAE) model rats after oral administration of YGP. The expression of cAMP response element binding protein (CREB) and growth-associated protein-43 (GAP-43) in SH-SY5Y cells treated with these six components, YGP extract, and YGP-containing serum were investigated to determine the pharmacodyamic material basis of YGP. Six bioactive components were detected in rat plasma, including songorine, benzoylhypaconitine, benzoylmesaconitine, neoline, karacoline and sweroside, which were rapidly absorbed after administration in EAE model rats.

Results: The main pharmacokinetic parameters of six bioactive components were determined, and the constituents increased CREB and GAP-43 expressions in serum-deprived SHSY5Y cells. The YGP-containing serum, six bioactive components, and YGP extract significantly increased the expression of both CREB and GAP-43 $(P<0.01)$, and there was no difference between the three groups.

Conclusion: The songorine, benzoylhypaconitine, benzoylmesaconitine, neoline, karacoline and sweroside were confirmed as the major bioactive components in YGP. The acquired data will be helpful for understanding the pharmacological and effective constituents of YGP.

Keywords: Yougui pill, experimental autoimmune encephalomyelitis, pharmacokinetics, pharmacology

\section{Introduction}

Yougui pill (YGP) is a classic Chinese medicine formula ${ }^{1}$ that has been used clinically for 400 years and is recorded in the Chinese Pharmacopoeia (2015 Edition). ${ }^{2}$ YGP is well known for its therapeutic effects on weakness of the waist in traditional Chinese medicine. With the development of research, YGP has recently been found to have neuroprotective effects on experimental autoimmune encephalomyelitis (EAE) in rats, ${ }^{3}$ an accepted animal model of multiple sclerosis (MS), which demonstrated the presence of axonal and neuronal damage.

MS is a multifocal demyelinating autoimmune disease of the central nervous system $(\mathrm{CNS}),{ }^{4}$ resulting in neurological disability in young adults. ${ }^{5}$ Promoting neuronal 
function and regeneration are important strategies for the treatment of MS. cAMP response element binding protein (CREB) has long been implicated in neuronal function, and the disruption of CREB function in the brain leads to nerve function damage. ${ }^{6}$ CREB is one of the key mediators of stimulus-induced nuclear responses that underlie the function of the nervous system. ${ }^{7}$ Much research indicates that CREB is a survival factor in various cellular models. ${ }^{8,9}$ Growth-associated protein-43 (GAP-43) is one of a small group of axonaltransported growth-associated proteins, and is required for axonal growth. ${ }^{10}$ Moreover, GAP-43 is an established marker for axonal regeneration, which is used for evaluating the regenerative capacity of the CNS. ${ }^{11,12}$ In previous research, it has been confirmed that GAP-43 is widely distributed and developmentally regulated in the CNS of rats. ${ }^{13}$ In the majority of MS white matter lesions, GAP-43 expression is decreased. ${ }^{14}$

As a complex prescription, YGP is composed of ten herbal medicines, ${ }^{15,16}$ including Rehmannia glutinosa (Gaertn.) DC., Aconitum carmichaeli var. carmichaeli, Cinnamomum cassia Presl., Cornus officinalis Siebold \& Zucc., Eucommia ulmoides Oliver., Dioscorea polystachya Turcz., Cuscuta chinensis Lam., Angelica sinensis (Oliv.) Diels., Lycium barbarum L. and colla cornus cervi. Some of the iridoids, flavones and alkaloids have been identified in individual herbs, such as catalpol, ${ }^{17}$ kaempferol $^{18}$ and fuziline. ${ }^{19}$ In our previous study, the HPLC-LTQ-Orbitrap$\mathrm{MS}^{\mathrm{n}}$ method and UPLC-QqQ-MS/MS method were developed to clarify the chemical profiling and quality evaluation of YGP. Eighty-three compounds were identified and 16 compounds were established for simultaneous determination in the YGP, including songorine, benzoylhypaconitine, benzoylmesaconitine, neoline, karacoline and sweroside, which have strong pharmacological activity. ${ }^{20-25}$

However, there are few reports focusing on the pharmacokinetic and pharmacological material basis of YGP. The development of a pharmacokinetic study and clarification of the pharmacological material basis of YGP for efficacy and safety research in clinical trials are in great demand. In this paper, YGP extract is used to investigate the neuroprotective effects of YGP via expression of CREB and GAP-43 in vitro. Then, we tried to detect 25 possible components of YGP in rat plasma, such as catalpol, kaempferol, rhmannioside A, loganin and cornuside I. Unfortunately, most of the components were not detected in rat plasma because of low content or strong metabolism. Six bioactive components were detected in rat plasma, including songorine, benzoylhypaconitine, benzoylmesaconitine, neoline, karacoline and sweroside. A ripid and accurate UPLC-ESI-MS/MS method was developed for quantitative determination of these components in rat plasma after oral administration of YGP. Finally, the neuroprotective effects of the six bioactive components were investigated for expression of CREB and GAP-43, and compared with other groups treated with YGP extract and YGP-containing serum in vitro.

\section{Materials and methods}

\section{Chemicals and materials}

YGP (batch number 4013596) was obtained from Tong Ren Tang Technologies Co., Ltd. (Beijing, China). The contents of songorine, benzoylhypaconitine, benzoylmesaconitine, neoline, karacoline and sweroside were detected by UPLCQqQ-MS/MS, and the contents of these components were $3.41 \mu \mathrm{g} / \mathrm{g}, 3.67 \mu \mathrm{g} / \mathrm{g}, 19.3 \mu \mathrm{g} / \mathrm{g}, 2.71 \mu \mathrm{g} / \mathrm{g}, 0.97 \mu \mathrm{g} / \mathrm{g}$ and 6.91 $\mu \mathrm{g} / \mathrm{g}$, respectively. All six standards were purchased from the Shaanxi Huayu Biotech Co., Ltd. (Xi'an, Shaanxi, China). Their purities were evaluated to be greater than $98.0 \%$ by HPLC-DAD. The reference standard of buspirone hydrochloride (Internal standard, IS) was purchased from Sigma (St Louis, MO, USA). Myelin basic protein (MBP) 68-86 (YGSLPQKSQRSQDENPV) was synthesized by Beijing SciLight Biotechnology Co. Ltd. (Beijing, China), and the purity was greater than $95.0 \%$. Incomplete Freund's adjuvant (IFA) and mycobacterium tuberculosis (MTB) were purchased from Difco Co. Ltd. (Franklin Lakes, NJ, US). Goat anti-rabbit IgG fluorescein isothiocyanate (FITC) and rabbit anti-goat IgG FITC were purchased from Zhongshan Golden Bridge (Beijing, China). CREB rabbit polyclonal antibody and GAP-43 goat polyclonal antibody were purchased from Santa Cruz Biotechnology, Inc. (Santa Cruz, CA, USA). HPLC-grade methanol and acetonitrile were both obtained from Fisher (Fair Lawn, NJ, USA). HPLC-grade formic acid was purchased from Merck (Darmstadt, Germany). HPLCgrade DMSO was purchased from Sigma (Sigma-Aldrich, MO, USA). Ultrapure water was produced by a Milli-Q Reagent Water System (Millipore, MA, USA).

\section{Pharmacokinetics}

Fourteen SPF-grade male Lewis rats, $220 \pm 20 \mathrm{~g}$ in weight, were purchased from Beijing Vital River Laboratories (China), and housed in the Experimental Animal Center of Capital Medical University (Beijing, China). The rats were divided into a control group and a model group $(n=7$ per 
group). All the animals had free access to food and water, and the indoor temperature and humidity were $23 \pm 2^{\circ} \mathrm{C}$ and $60 \pm 5 \%$, respectively. The animals were housed under the above conditions for a week before the start of the experiment. For establishment of the EAE model, $5 \mathrm{mg} \mathrm{MBP}{ }_{68-86}$ was dissolved in $10 \mathrm{~mL}$ normal saline, and mixed with 10 mL IFA and $2 \mathrm{mg}$ MTB as antigen. Then, $200 \mu \mathrm{L}$ antigen was injected subcutaneously into 2 hind footpads of rats, as previously described. ${ }^{26}$

In the pharmacokinetic study, $0.5 \%$ CMC-Na solution was used for solubilizing YGP. A dose of $2500 \mathrm{mg}$ / $\mathrm{kg}$ YGP was administered i.g. in the model group, and the control group was administered $0.5 \%$ CMC-Na solution. Then, aliquots of $0.3 \mathrm{~mL}$ blood samples were obtained from the jugular vein of rats in the two groups according to a specific schedule (pre-dose and 0.083, $0.167,0.25,0.33,0.50,1,2,4,6,8,12$ and $24 \mathrm{hrs})$. The blood was collected in heparinized $1.5 \mathrm{~mL}$ polythene tubes and separated by centrifugation at $5,000 \mathrm{~g}$ for 10 mins. Then, the plasma was stored at $-80^{\circ} \mathrm{C}$ until analysis.

Pharmacokinetic parameters including the biological half-life $\left(t_{1 / 2}\right)$, maximum plasma time $\left(T_{\max }\right)$, maximum concentration $\left(\mathrm{C}_{\max }\right)$, concentration-time curve from time zero to the last measurable plasma concentration point and time infinity ( $\mathrm{AUC}_{0 \text {-last }}$ and $\mathrm{AUC}_{\text {Inf }}$ ), mean residence time (MRT) and clearance $(\mathrm{Cl})$ were calculated using a noncompartmental method with drug and statistics (DAS) software, version 2.0 (Shanghai, China). All the results were expressed as arithmetic mean \pm standard deviation (SD).

All procedures were in accordance with the National Institute of Heath's guidelines regarding the principles of animal care (2004). Furthermore, the animal facilities and protocols were approved by the Animal Care and Use Committee of Capital Medical University (License number: AEEI-2015-060; Date of approval: May 8, 2015; Beijing, China).

\section{LC-MS/MS conditions}

Analysis was performed on a Shimadzu UPLC system (Shimadzu, Kyoto, Japan) coupled with a binary solvent delivery system, an automatic injector, a column oven, a DAD detector and a workstation. An Agilent Zorbax XDB C18 reverse phase column $(50 \mathrm{~mm} \times 2.1 \mathrm{~mm}, 3.5 \mu \mathrm{m})$ was used to separate samples. Mobile phase A (water containing $0.1 \%$ formic acid) and phase B (methanol containing $0.1 \%$ formic acid) were utilized in gradient elution mode. The gradient elution program was as follows: $0.00-0.60 \mathrm{mins}$, $2 \%$ B; $0.60-3.00$ mins, $2-45 \%$ B; $3.00-4.50$ mins, $45-85 \%$ B; $4.50-4.51 \mathrm{mins}, 85-2 \% \mathrm{~B}$; and $4.51-6.00 \mathrm{mins}, 2 \% \mathrm{~B}$. The flow rate was $0.3 \mathrm{~mL} / \mathrm{mins}$, and a $10 \mu \mathrm{L}$ sample was injected.

The UPLC system was connected to an API 4000 Qtrap mass spectrometer (Applied Biosystems/MDS Sciex, Concord, ON, Canada) via an ESI interface. Samples were analyzed in positive ion mode and monitored in multiple reaction monitoring (MRM) mode. The curtain (CUR), nebulizer (Gas 1) and heater (Gas 2) gases used high purity nitrogen, and the flow rates were set at 20,55 and $55 \mathrm{psi}$, respectively. The source temperature was set at $500^{\circ} \mathrm{C}$ and the ion spray needle voltage was $5500 \mathrm{~V}$. The MS parameters of the six bioactive components were shown in Table 1.

\section{Method validation}

The method validation included selectivity, linearity, lower limit of quantification (LLOQ), accuracy, precision, recovery, matrix effect and stability. The selectivity was validated by comparing the blank plasma, standard-spiked plasma and experimental rat plasma. The linearity was constructed by least squares linear regression. The ratios of peak area of reference substance to IS $(y)$ were regressed against the corresponding concentration $(x)$ with a weighting factor of $1 / x^{2}$. The LLOQ was defined as the lowest concentration, which was serial dilution on the calibration curve with acceptable precision and

Table I Main analytical parameters of 6 compounds determined by ultra-performance liquid chromatography coupled with triplequadrupole tandem mass spectrometry

\begin{tabular}{|l|l|l|l|l|l|}
\hline Compound & Parent ion $(\mathbf{m} / \mathbf{z})$ & Product ion for quantification $(\mathbf{m} / \mathbf{z})$ & DP $^{\mathbf{a}}(\mathbf{V})$ & $\mathbf{C E}^{\mathbf{b}}(\mathbf{e V})$ & $\mathbf{C X P}^{\mathbf{c}}(\mathbf{V})$ \\
\hline songorine & 358.2 & 340.2 & 98 & 35 & 20 \\
benzoylhypaconitine & 547.2 & 542.2 & 150 & 41 & 15 \\
benzoylmesaconitine & 590.2 & 540.2 & 70 & 47 & 30 \\
neoline & 438.2 & 420.2 & 60 & 40 & 31 \\
karacoline & 378.2 & 360.2 & 60 & 42 & 25 \\
sweroside & 381.1 & 219.2 & 130 & 25 & 15 \\
\hline
\end{tabular}

Abbreviations: DP, declustering potential; CE, collision energy; CXP, cell exit potential. 
accuracy. The precision and accuracy were validated by performing replicate QC samples. The QC samples were set as three different concentrations $(40,10$ and $1 \mathrm{ng} / \mathrm{mL})$; each concentration was quantitative determination every single day as intra-day variation. Then the QC samples were calculated in three consecutive days as inter-day variation. The recovery was validated by comparing the peak areas of analyte-added plasma and true concentration of standards. The matrix effect was calculated by comparing the peak areas of standards in blank plasma and the corresponding peak areas in methanol. The stability was evaluated from different aspects, including freeze-thaw stability $\left(-20^{\circ} \mathrm{C}\right.$, three cycles), short-term stability $\left(20^{\circ} \mathrm{C}\right.$ for $24 \mathrm{hrs})$ and long-term stability $\left(20^{\circ} \mathrm{C}\right.$ for $\left.72 \mathrm{hrs}\right)$.

\section{Sample preparation of pathology and ultrastructural}

For hematoxylin eosin (HE) staining, the rats were anesthetized with $1 \%$ pentobarbital sodium solution (w/v, $40 \mathrm{mg} / \mathrm{kg}$ ); then, the chest was opened and the heart was exposed. A catheter was inserted into the left ventricle, and the right atrial appendage was cut with eye scissors. Normal saline $(250 \mathrm{~mL})$ was perfused in rats by a peristaltic pump, followed by $250 \mathrm{~mL}$ of $4 \%$ paraformaldehyde. The brains and spinal cords were collected and fixed in $4 \%$ paraformaldehyde for $72 \mathrm{hrs}$.

For transmission electron microscopy (TEM), the rats were anesthetized and dissected in the same method as with HE staining. Then, $250 \mathrm{~mL}$ of normal saline was perfused in rats by a peristaltic pump, followed by 250 $\mathrm{mL}$ of $2 \%$ paraformaldehyde and $2 \%$ glutaraldehyde. The cerebral white matter and lumbar region of the spinal cord were cut into one cubic milliliter sections and fixed in 3\% glutaraldehyde, following three rinses in PBS.

\section{Pathology and ultrastructural observations}

The sections of the cerebral white matter and the lumbar region of spinal cord samples were fixed and embedded in paraffin wax. The sections (thickness, $5 \mu \mathrm{m}$ ) of the paraffin-embedded tissues were stained with hematoxylin eosin, and examined under light microscope (Nikon Eclipse 80i). The inflammatory cell infiltration was detected with an image analysis system, and the evaluation methodology was described as follows: 0 , no infiltrates; 1 , sporadic inflammatory cells; 2, single inflammatory cells were detected around blood vessels; 3, large number of inflammatory cells were detected around blood vessels; and 4 , perivascular cuff formation or diffuse infiltration of inflammatory cells were detected around blood vessels. ${ }^{27}$

TEM was used for observing the morphology of axons and myelin in cerebral white matter and the spinal cord. The samples were stained by uranyl acetate and alkaline lead citrate and observed in TEM. The images were analyzed using Image-Pro Plus 6.0 (Media Cybernetics, Inc., Washington, USA), and the mean area/diameter was used for evaluating the lesion degree of myelin. ${ }^{28}$

\section{Cells culture and treatment}

SH-SY5Y cells (CRL-2266) were obtained from the American Type Culture Collection (CRL-2266), and cultured in RPMI 1640 supplemented with 10\% (FBS; Gibco Invitrogen Corporation, Carlsbad, CA, USA), 1\% double antibiotics and $10 \mathrm{mmol} / \mathrm{L}$ HEPES at $37^{\circ} \mathrm{C}$ in a humidified atmosphere of $5 \% \mathrm{CO}_{2}$. Cells were passed once every 3 days. $^{29}$

\section{YGP-containing serum preparation}

Another 20 SPF-grade male Lewis rats $(220 \pm 20 \mathrm{~g})$ were divided into a normal group $(\mathrm{n}=10)$ and a YGP group $(\mathrm{n}=10)$, and were intragastrical administered distilled water and 2,500 mg/kg/day YGP for 7 days, respectively. A half hour after the last administration, rats were anesthetized with $1 \%$ pentobarbital sodium solution (w/v, $40 \mathrm{mg} /$ $\mathrm{kg}$ ). Blood was sterilely collected through the ventral aorta. After settling for $2 \mathrm{hrs}$ at room temperature, the blood samples were centrifuged at $3,000 \mathrm{r} / \mathrm{min}$ at $4^{\circ} \mathrm{C}$ for 15 mins, and inactivated at $56^{\circ} \mathrm{C}$ for 30 mins. The samples were stored at $-80^{\circ} \mathrm{C}$ after being filtered through a microfiltration membrane $(0.22 \mu \mathrm{m})$.

\section{Measurement of cell viability}

The SH-SY5Y cells were plated at a density of $2 \times 10^{3}$ cells/ well in a 96-well plate to adhere overnight and then treated with $0.25 \%$ FBS for $24 \mathrm{hrs}$ to establish the model of axonal outgrowth inhibition, which was followed by incubating with various concentrations of YGP-containing serum $(0.1 \%, 0.25 \%, 0.5 \%, 1 \%, 2.5 \%, 5 \%, 10 \%)$ for $24 \mathrm{hrs}$. After treatment, $20 \mu \mathrm{L}$ of MTT solution $(5 \mathrm{mg} / \mathrm{mL})$ was added and incubated for $4 \mathrm{hrs}$ at $37^{\circ} \mathrm{C}$. The supernatant was removed and $150 \mu \mathrm{L}$ of DMSO/well was added to dissolve the intracellular crystalline formazan product. Cell viability was determined by measuring the absorbance at $490 \mathrm{~nm}$ using a SpectraMax Plus 384 Microplate Reader (Molecular 

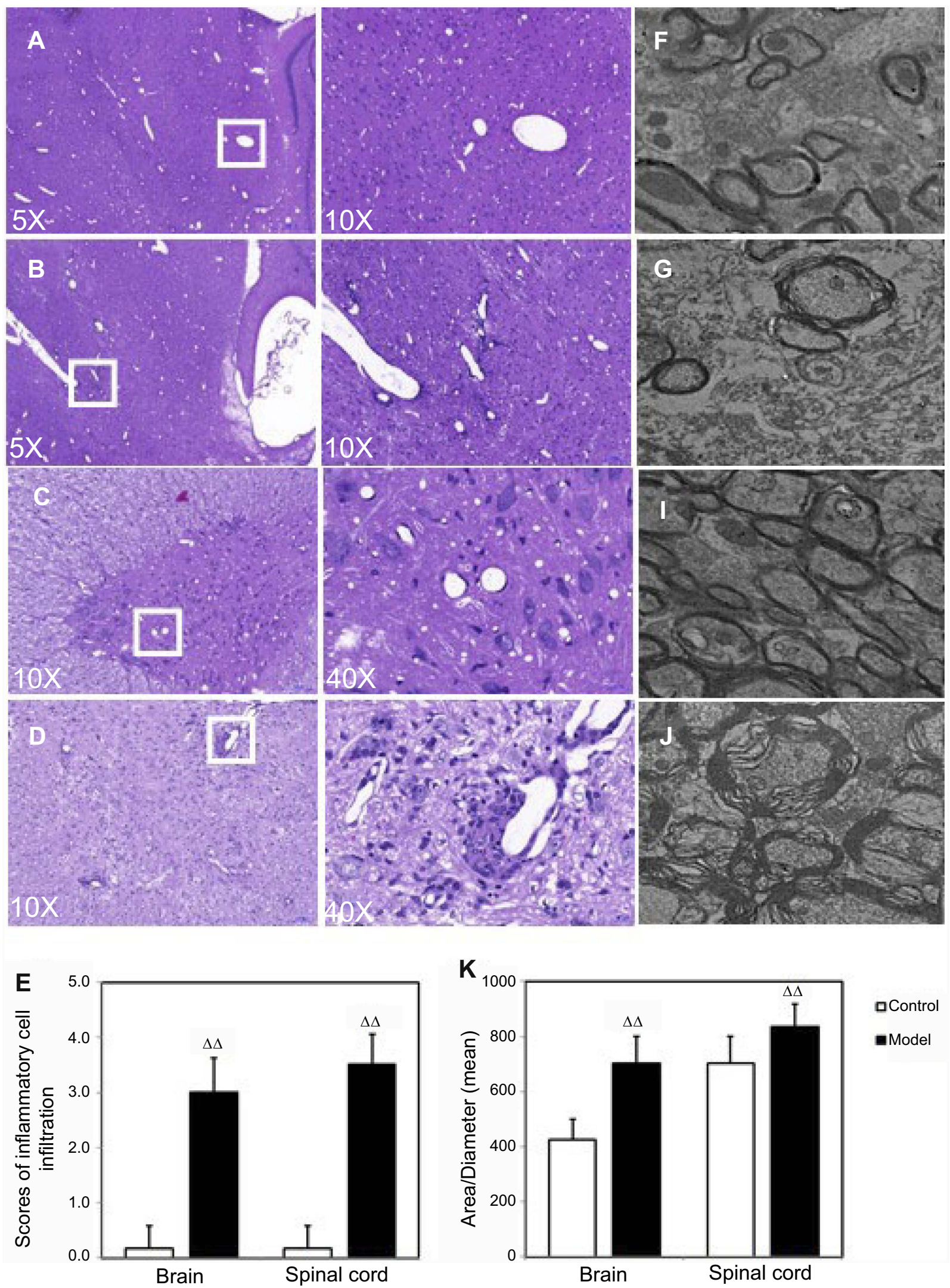

Figure I Pathology and ultrastructural observations in experimental autoimmune encephalomyelitis (EAE) model rats. Figures A-D show histological changes in the brains and spinal cords of the EAE model rats, $n=7$. (E) The inflammatory cell infiltration was assessed by the scores. ${ }^{\Delta \Delta} P<0.01$ vs control group. ( $\left.F-I\right)$ Ultrastructural changes in the myelin sheaths of the brains and spinal cords of the EAE model rats. Scale bars: magnification: $6000 \times, n=10$. (J) The ultrastructural changes were assessed by the ratios of area/diameter of myelin sheaths. ${ }^{\Delta \Delta} P<0.0$ I vs control group. 
Table 2 The linearities, ranges and LLOQs of the six bioactive components in rat plasma determined by ultra-performance liquid chromatography coupled with triple-quadrupole tandem mass spectrometry

\begin{tabular}{|l|l|l|l|l|}
\hline Analyte & Calibration curve & $\mathbf{R}^{\mathbf{2}}$ & Range (ng/mL) & Lower Limit of Quantification (ng/mL) \\
\hline songorine & $y=0.2460 x-0.01610$ & 0.9958 & $0.50-50.0$ & 0.5 \\
benzoylhypaconitine & $y=0.0406 x-0.00628$ & 0.9956 & & \\
benzoylmesaconitine & $y=0.0265 x-0.00152$ & 0.9964 & & \\
neoline & $y=0.0638 x-0.00335$ & 0.9986 & & \\
karacoline & $y=0.0991 x-0.00770$ & 0.9951 & & \\
sweroside & $y=0.0135 x-0.00062$ & 0.9974 & & \\
\hline
\end{tabular}

Table 3 Main pharmacokinetic parameters of six bioactive components in experimental autoimmune encephalomyelitis (EAE) model rats after oral administration of Yougui pill $\left(n=7\right.$, median $\pm S D$ for $t_{\max }$, mean $\pm S D$ for other parameters)

\begin{tabular}{|c|c|c|c|c|c|c|c|}
\hline Parameters & Unit & Songorine & Benzoylhypaconitine & Benzoylmesaconitine & Neoline & Karacoline & Sweroside \\
\hline$t_{1 / 2}$ & $\mathrm{~h}$ & $2.53 \pm 0.63$ & $1.67 \pm 0.23$ & $2.11 \pm 0.50$ & $1.82 \pm 0.33$ & $2.12 \pm 0.88$ & $3.32 \pm 1.51$ \\
\hline $\mathrm{T}_{\max }$ & $\mathrm{h}$ & $1.00 \pm 0.09$ & $0.33 \pm 0.09$ & $0.33 \pm 0.25$ & $0.50 \pm 0.30$ & $0.50 \pm 0.32$ & $1.00 \pm 0.63$ \\
\hline $\mathrm{C}_{\max }$ & $\mathrm{ng} / \mathrm{mL}$ & $1 \mathrm{I} .7 \pm 5.27$ & $18.5 \pm 4.28$ & $29.2 \pm 7.49$ & $97.6 \pm 46.7$ & $34.1 \pm 9.20$ & $232 \pm 73.5$ \\
\hline$A \cup C_{0 \text {-last }}$ & $\mathrm{ng} \mathrm{h} / \mathrm{mL}$ & $52.2 \pm 23.2$ & $47.5 \pm 15.5$ & $67.6 \pm 25.1$ & $315 \pm 153$ & $88.1 \pm 26.5$ & $1115 \pm 614$ \\
\hline$A \cup C_{\operatorname{lnf}}$ & $\mathrm{ng} \mathrm{h} / \mathrm{mL}$ & $55.3 \pm 25.1$ & $49.8 \pm 16.1$ & $73.6 \pm 28.5$ & $320 \pm 155$ & $96.6 \pm 29.5$ & $1139 \pm 619$ \\
\hline $\mathrm{Cl}$ & $\mathrm{L} / \mathrm{h} / \mathrm{kg}$ & $51.1 \pm 4.92$ & $28.0 \pm 5.5 I$ & $16.8 \pm 3.01$ & $3.07 \pm 0.92$ & $0.35 \pm 0.07$ & $1.48 \pm 0.13$ \\
\hline MRT & $\mathrm{h}$ & $4.16 \pm 0.64$ & $2.76 \pm 0.29$ & $3.06 \pm 0.47$ & $2.74 \pm 0.4 I$ & $3.13 \pm 1.02$ & $4.33 \pm 1.19$ \\
\hline
\end{tabular}

Abbreviations: AUC, Area Under Curve; $\mathrm{Cl}$, clearance rate; MRT, Mean Retention Time.

Devices, Sunnyvale, CA, USA). The optical density of normal cells was defined as $100 \%$.

\section{High content analysis}

SH-SY5Y cells were plated at a density of $2 \times 10^{3}$ cells/well in a 96-well plate and allowed to adhere overnight. Then, they were treated with $0.25 \%$ FBS for $24 \mathrm{hrs,} \mathrm{followed} \mathrm{by} \mathrm{treat-}$ ments as described in 2.3 or $5 \%$ YGP-containing serum, mixed standards (the final concentrations were as follows: $20.8 \mathrm{ng} / \mathrm{mL}$ of songorine, $17.0 \mathrm{ng} / \mathrm{mL}$ of benzoylhypaconitine, $31.4 \mathrm{ng} / \mathrm{mL}$ of benzoylmesaconitine, $169.0 \mathrm{ng} / \mathrm{mL}$ of neoline, $42.0 \mathrm{ng} / \mathrm{mL}$ of karacoline and $302.0 \mathrm{ng} / \mathrm{mL}$ of sweroside) and YGP extract (the final concentration was $19.0 \mu \mathrm{g} / \mathrm{mL}$ ). Afterward, the cells were fixed with $4 \%$ paraformaldehyde at room temperature for 30 mins and rinsed with PBS, followed by permeabilization for $10 \mathrm{mins}$ in $0.1 \%$ Triton-X 100, and then blocked by $3 \%$ BSA. Then, SH-SY5Y cells in a $96-w e l l$ plate were incubated with anti-CREB (1:50) and anti-GAP43 (1:50) at $4^{\circ} \mathrm{C}$ for $18 \mathrm{hrs}$, respectively. After washing, the cells were incubated with goat anti-rabbit IgG FITC or rabbit antigoat IgG FITC at room temperature for $1 \mathrm{hr}$ and counterstained with DAPI for 5 mins. Automated cell image acquisition was performed on a Thermo Fisher Scientific Cellomics ArrayScan VTI High Content Screening Reader using a 10 objective. DAPI (representing the nuclei) and FITC (representing the objective protein) were measured in channels 1 and
2, respectively. Image analysis was done using the Compartmental Analysis Bioapplication (Thermo Fisher Scientific). Image acquisition was set to six fields per well. Cell number was evaluated by measuring the number of DAPI staining cells ("Selected Valid Object Count") in Channel 1. FITC staining was measured ("MEAN_Target Total Inten Ch2") in Channel 2. The results were expressed as a percentage of the average intensity of control group cells. ${ }^{30}$

\section{Data analysis}

The pharmacokinetic parameters were calculated by noncompartmental method using DAS Software (version 2.0, China State Drug Administration, Shanghai, China). Statistical analysis was performed on SPSS version 17.0 (International Business Machines Corporation, New York, USA). Significant differences between group-values were analyzed by one-way ANOVA followed by least significant difference or Tambane's T2 analysis. Data were expressed as the means $\pm \mathrm{SD}$, and $P<0.05$ were statistically significant.

\section{Results}

\section{Pathology and ultrastructural observations in EAE model rats}

In cerebral white matter and lumbar region of the spinal cord, the scores of inflammation significantly increased in 

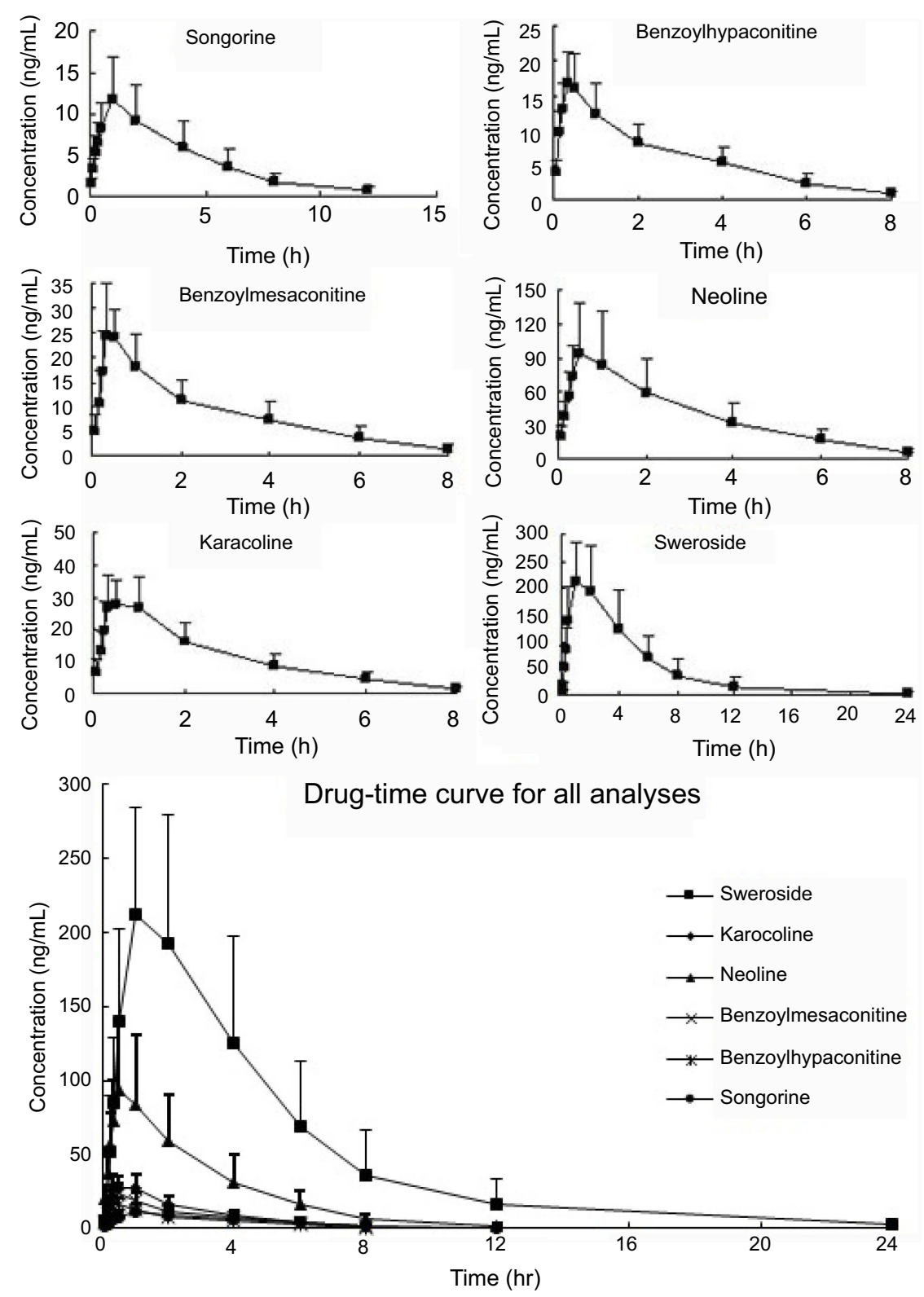

Figure 2 Mean drug-time profile of target compounds determined by ultra-performance liquid chromatography coupled with triple-quadrupole tandem mass spectrometry method after oral administration of $2500 \mathrm{mg} / \mathrm{kg}$ Yougui pill to rats $(\mathrm{n}=7)$.

the model group compared with the control group $(P<0.01)$ (Figure 1). Increased ratios were found in the brain and spinal cord of the model group compared to the control group $(P<0.01)$ (Figure 1).

\section{LC-MS/MS method validation}

According to the international guidelines ${ }^{31,32}$ and the established procedures in our lab, ${ }^{33-36}$ the method was validated as follows: selectivity, linearity, LLOQ, accuracy, precision, recovery, matrix effect and stability. With validation of selectivity, there was no potential endogenous interference in the plasma. All of the six components showed good linearity in a wide range, and the LLOQ was $0.50 \mathrm{ng} / \mathrm{mL}$ for these components (Table 2). The intra- and inter-batch precisions and accuracies of the six components were less than $15 \%$ within the range between $85 \%$ and $115 \%$. The recovery, stability and matrix effect were also within the acceptable range of international guidelines. The method validation was published in our previous study. $^{37}$

\section{Pharmacokinetics}

A simple, sensitive and accurate LC-ESI-MS/MS method was successfully developed and applied in the pharmacokinetic studies of the six bioactive components in YGP 


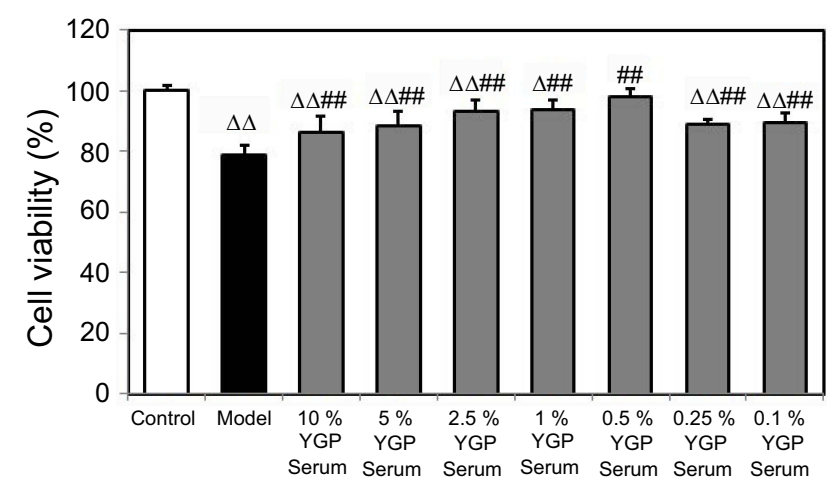

Figure 3 Yougui pill (YGP)-containing serum protected SH-SY5Y cells in the model of axonal outgrowth inhibition. SH-SY5Y cells were treated by serum deprivation followed with YGP serum for $24 \mathrm{hrs,} \mathrm{respectively,} \mathrm{and} \mathrm{assessed} \mathrm{later.} \mathrm{Cells} \mathrm{were}$ assessed by MTT assay and optical density of control cells was defined as $100 \%$. Data represent the mean \pm SD. ${ }^{\Delta} P<0.05,{ }^{\Delta \Delta} P<0.01$ vs control group cells; ${ }^{\Delta} P<0.01$ vs model group cells; Data were analyzed by one-way ANOVA followed by least significant difference, $n=4$ per group.

(songorine, benzoylhypaconitine, benzoylmesaconitine, neoline, karacoline and sweroside) in Lewis model rats after oral administration of $2,500 \mathrm{mg} / \mathrm{kg}$ of YGP. PK parameters for the six compounds are shown in Table 3. Mean plasma concentration-time profiles for these six compounds are shown in Figure 2.

\section{YGP-containing serum protected $\mathrm{SH}$ - SY5Y cells in the model of axonal outgrowth inhibition}

In order to evaluate cell injury in SH-SY5Y cells induced by low serum medium $(0.25 \%$ FBS $)$ and the possible cytoprotective effect of YGP-containing serum, we assessed treatment with different concentrations of YGP-containing serum $(0.1 \%$ YGP serum+9.9\% normal serum, $0.25 \%$ YGP serum $+9.75 \%$ normal serum, $0.5 \%$ YGP serum $+9.5 \%$ normal serum, 1\% YGP serum+9\% normal serum, 2.5\% YGP serum $+7.5 \%$ normal serum, 5\% YGP serum $+5 \%$ normal serum and 10\% YGP serum) after treatment with low serum medium $(0.25 \%$ FBS $)$ by using the MTT assay. As shown in Figure 3, serum deprivation resulted in a significant reduction of cell viability $(P<0.01)$, whereas treatment with YGP-containing serum protected SH-SY5Y cells $(P<0.01)$.

\section{YGP-containing serum increased CREB and GAP-43 expressions in serum- deprived SH-SY5Y cells}

The results demonstrated that serum deprivation suppressed CREB expressions $(P<0.01)$, and $5 \%$ and $0.5 \%$ YGP- containing serum treatment significantly increased this expression $(P<0.01)$ (Figure 4$)$. Compared with untreated cells, serum deprivation downregulated GAP-43 expression $(P<0.01)$. Likewise, GAP-43 was further increased when treated with $1 \%, 0.5 \%$ and $0.25 \%$ YGP-containing serum $(P<0.01)$ (Figure 5).

\section{YGP extract increased CREB and GAP-43 expressions in serum-deprived SH-SY5Y cells}

The results demonstrated that serum deprivation suppressed CREB expressions $(P<0.01)$, and mixed standards and YGP extract treatment significantly increased this expression $(P<0.01)$ (Figure 6). Compared with untreated cells, serum deprivation downregulated GAP-43 expression $(P<0.01)$ and GAP-43 was further increased when treated with mixed standards and YGP extract $(P<0.01)$ (Figure 7). There was no difference between the 1\% YGPcontaining serum, mixed standards and YGP extract regarding CREB and GAP-43 expressions.

\section{Discussion}

In the light microscopic examination of HE-stained cerebral white matter and lumbar region of the spinal cord, pathological changes were clearly observed in the tissue of model group rats. Numerous inflammatory cells were observed surrounding the small veins in model group rats. In the TEM analysis, myelin sheaths of the brain and spinal cord were loose and disintegrated, which was observed in both the cerebral white matter and spinal cord in model group rats. The ratios of area/diameter of the myelin sheaths were used for estimating the axonal demyelination and damage.

Both positive ion mode and negative ion mode were evaluated for all six components and IS. The $[\mathrm{M}+\mathrm{H}]^{+}$or $[\mathrm{M}$ $+\mathrm{Na}]^{+}$ions of standards showed higher abundances than those in negative ion mode. The precursor ion $[\mathrm{M}+\mathrm{H}]^{+}$ found at $\mathrm{m} / \mathrm{z}=358.2,574.2,590.2,438.2,378.2$ and 386.1 for songorine, benzoylhypaconitine, benzoylmesaconitine, neoline, karacoline and IS, respectively. The precursor ion of sweroside was an adduct ion $[\mathrm{M}+\mathrm{Na}]^{+}$, which was found at $\mathrm{m} / \mathrm{z}=381.1$. Other conditions had also been further optimized, such as CUR, Gas 1, Gas 2, capillary temperature and collision energy.

The LC-ESI-MS/MS method has been fully validated in terms of selectivity, LLOQ, accuracy, precision, recovery, matrix effect and stability. Pharmacokinetic parameters including the biological half-life $\left(t_{1 / 2}\right)$, maximum plasma 

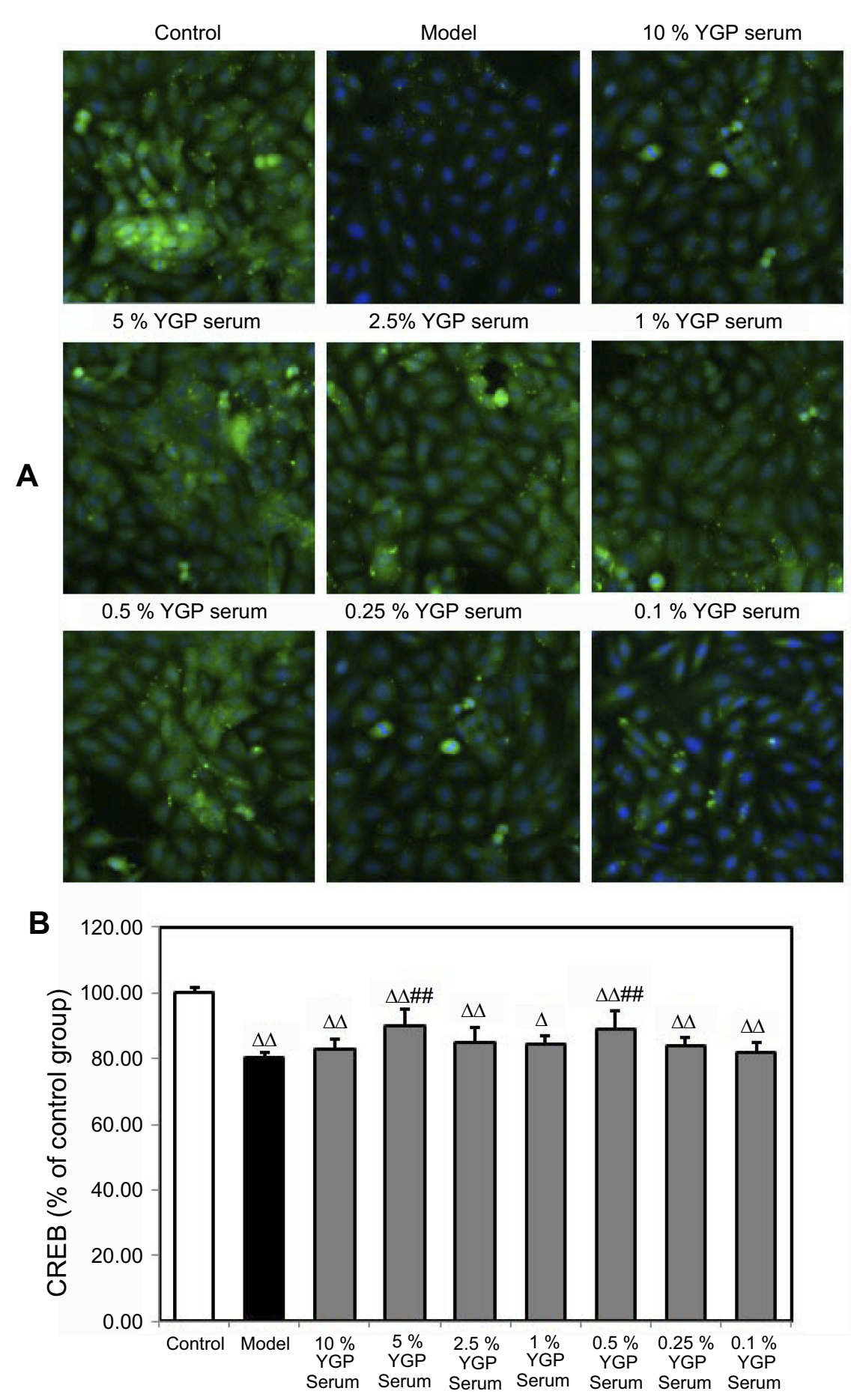

Figure 4 Yougui pill (YGP) serum increased CREB expression in serum-deprived SH-SY5Y cells. (A) High content analysis of CREB treated by serum deprivation followed with YGP-containing serum for $24 \mathrm{hrs}$, respectively, and assessed later. Images show immunostaining of SH-SY5Y cells (I0X magnification). (B) Results of CREB are expressed as the mean \pm SD indicated as percentage of the respective normal cells and analyzed using one-way ANOVA with Least-Significant Difference (LSD) analysis followed by least significant. ${ }^{\Delta} P<0.05,{ }^{\Delta \Delta} P<0.0$ I vs control group cells; ${ }^{\# \#} P<0.0$ I vs model group cells. $n=4$ per group.

time $\left(\mathrm{T}_{\max }\right)$ and concentration $\left(\mathrm{C}_{\mathrm{max}}\right)$, mean area under the plasma concentration-time curve from time zero to the last measurable plasma concentration point and time infinity $\left(\mathrm{AUC}_{0 \text {-last }}\right.$ and $\left.\mathrm{AUC}_{\text {Inf }}\right)$, clearance $(\mathrm{Cl})$ and mean residence time (MRT) were observed. It appears that after a single oral administration of YGP, the analytes had short halflives, and all the $\mathrm{T}_{\max }$ were less than $1 \mathrm{hr}$. It indicated that their absorption and distribution were rapid in blood. Except for sweroside, the analytes were eliminated in less than $12 \mathrm{hrs}$. It indicated that YGP should be multi-dosed orally 


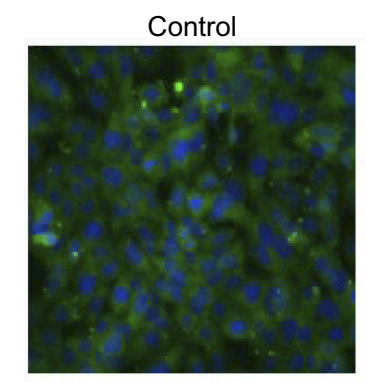

$5 \%$ YGP serum

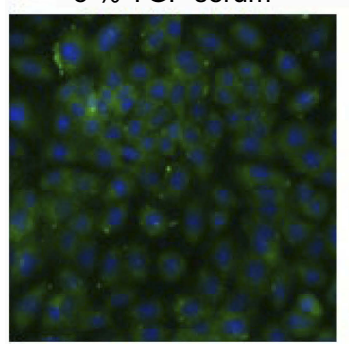

$0.5 \%$ YGP serum
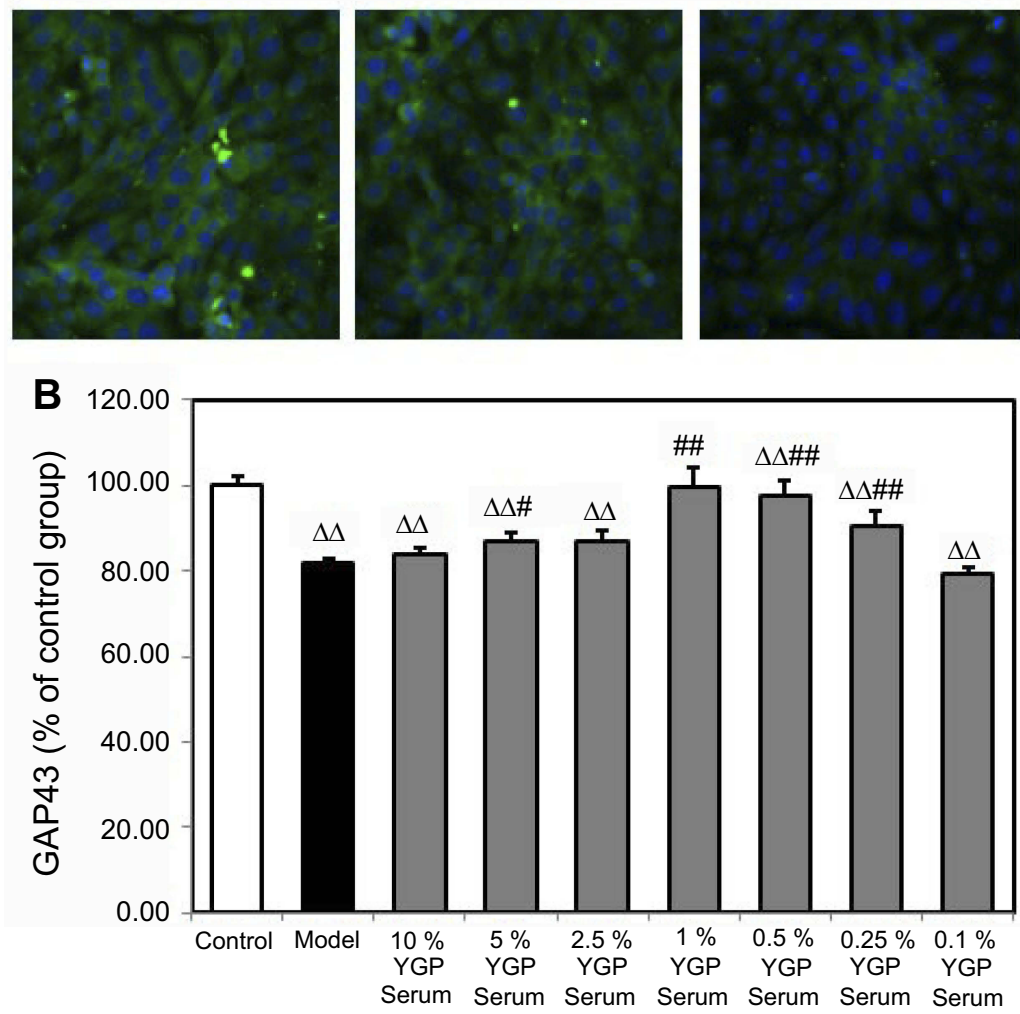

Figure 5 Yougui pill (YGP) serum increased GAP-43 expression in serum-deprived SH-SY5Y cells. (A) High content analysis of GAP-43 treated by serum deprivation followed with YGP-containing serum for 24 hrs, respectively, and assessed later. Images show immunostaining of SH-SY5Y cells (I0X magnification). (B) Results of GAP43 are expressed as the mean \pm SD indicated as percentage of the respective normal cells and analyzed using one-way ANOVA with Least-Significant Difference (LSD) analysis followed by least significant. ${ }^{\Delta \Lambda} P<0.01$ vs control group cells; ${ }^{\#} P<0.01$, ${ }^{\#} P<0.05$ vs model group cells. $n=4$ per group.

in a relative short period. Through analysis of $\mathrm{C}_{\max }$, the analytes showed low concentrations in plasma, which may indicate that the pharmacological effects of YGP originated from the superposition effects after multiple dosing.

Promoting neuronal function and regeneration are important strategies for the treatment of MS. CREB and
GAP-43 are two key factors, which contribute neuronal function and axon growth. ${ }^{7,10}$ In our preliminary study, it has been confirmed that YGP had a therapeutic action in EAE rats. ${ }^{26}$ It was found that YGP could abrogate demyelination and axonal damage and inhibit NogoA, NgR and RhoA expression in rats of EAE. ${ }^{38,39}$ Furthermore, YGP 

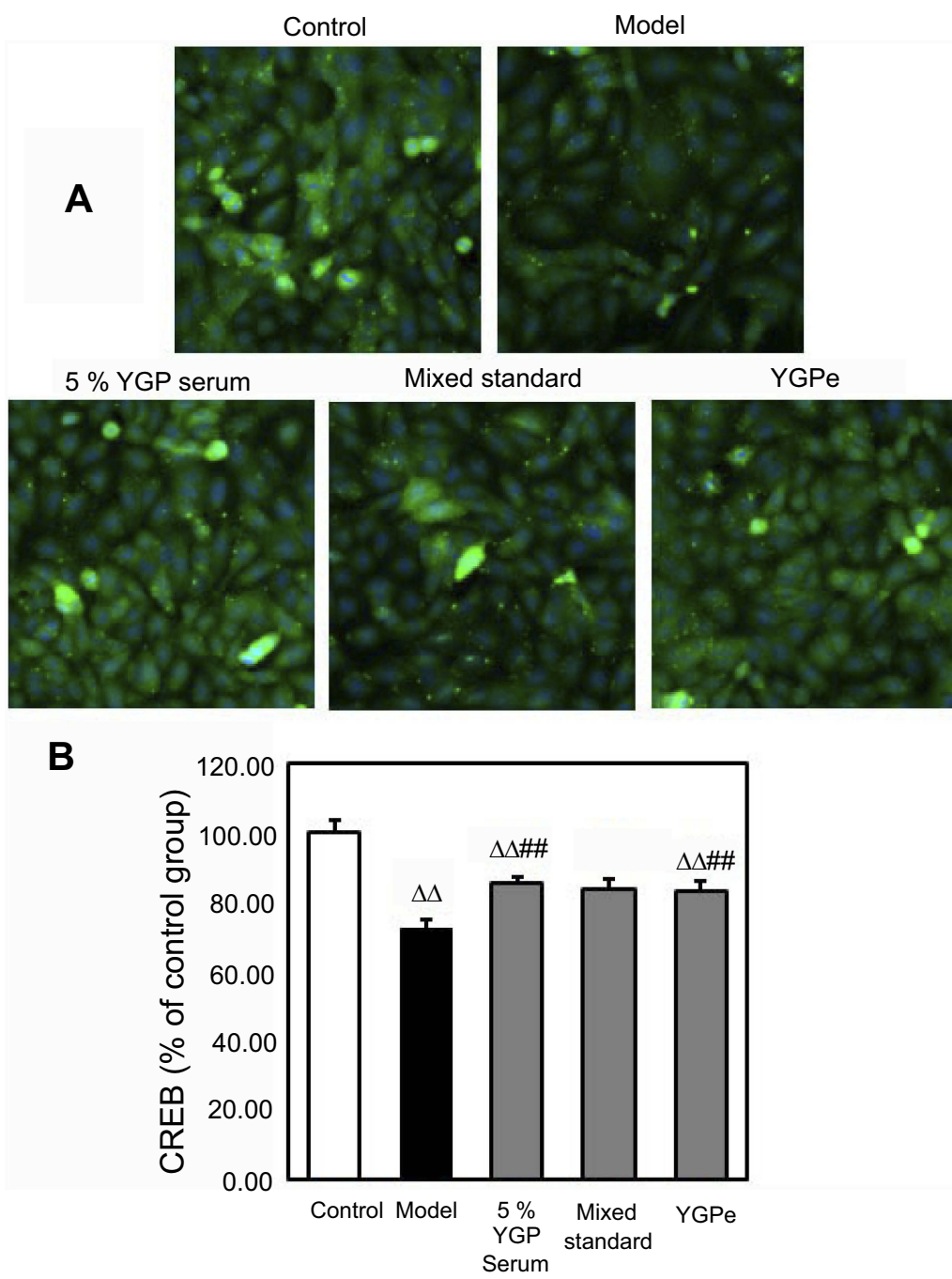

Figure 6 Yougui pill (YGP) extract increased CREB expression in serum-deprived SH-SY5Y cells. (A) High content analysis of CREB treated by serum deprivation followed with $5 \%$ YGP-containing serum, mixed standards and YGP extract for $24 \mathrm{hrs}$, respectively, and assessed later. Images show immunostaining of SH-SY5Y cells (I0X magnification). (B) Results of CREB are expressed as the mean \pm SD indicated as percentage of the respective normal cells and analyzed using one-way ANOVA with Least-Significant Difference (LSD) analysis followed by least significant. ${ }^{\Delta \Lambda} P<0.0$ I vs control group cells; ${ }^{\# \#} P<0.01$ vs model group cells. $n=4$ per group.

also could exhibit a neuroprotective effect on promoting nerve regeneration in EAE mice. ${ }^{3}$ In this study, YGPcontaining serum treatment significantly increased expression of CREB and GAP-43. It indicated that YGP had a therapeutic action on MS, and the mechanism was to increase expression of CREB and GAP-43.

In this study, the effect targets and basic effective material of YGP were further studied via in vitro experiment. A comprehensive analysis was applied for the component analysis of YGP in our previous research, including qualitative and quantitative assessments. Eighty-three compounds have been identified or tentatively identified in YGP, and 25 reference standards were availably purchased. In this study, 25 compounds were searched for in plasma. As a result, only songorine, benzoylhypaconitine, benzoylmesaconitine, neoline, karacoline and sweroside were detected, which were identified as prototypes in plasma.

According to these results of pharmacokinetics, we hypothesized the six components in plasma were the pharmacodynamic material basis of YGP, and could promote nerve regeneration. Therefore, the effect of the YGP-containing serum, six components and YGP extract were compared in a model of axonal outgrowth inhibition in vitro. YGP extract was used to investigate the neuroprotective effects via expression of CREB and GAP-43 in vitro. The effects of mixed standards and YGP extract on CREB and GAP-43 of serum-deprived SH-SY5Y cells were observed. In addition, the difference between the 5\% YGP-containing serum, mixed standards and YGP extract on CREB and GAP-43 were also investigated. As 


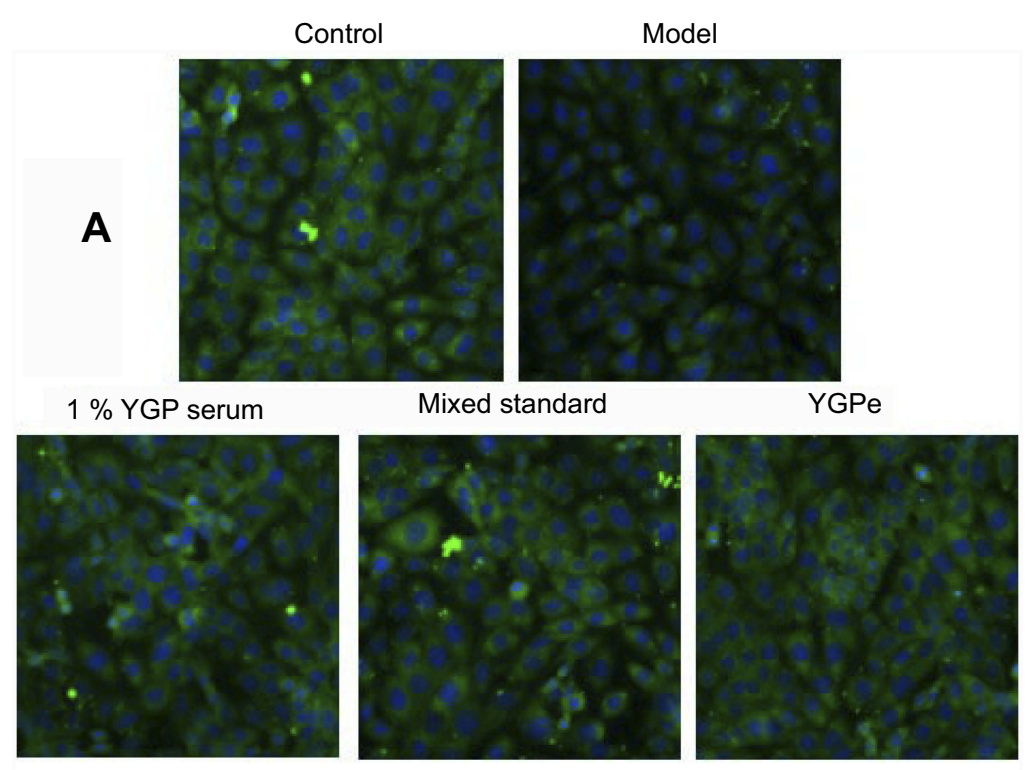

B

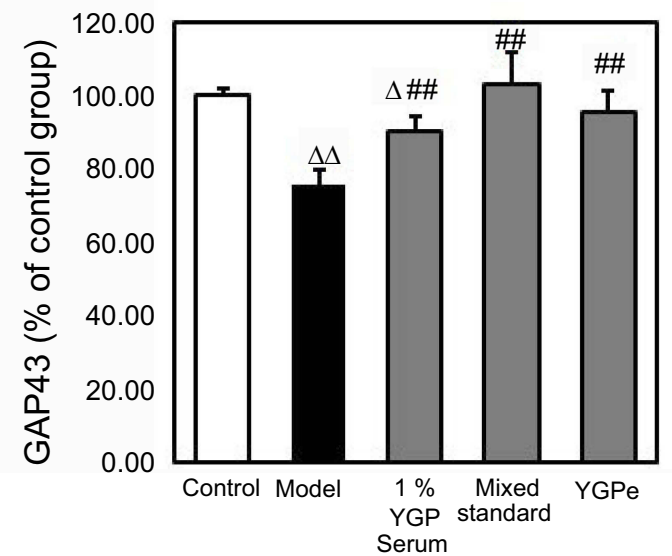

Figure 7 Yougui pill (YGP) extract increased GAP-43 expression in serum-deprived SH-SY5Y cells. (A) High content analysis of GAP-43 treated by serum deprivation followed with I\% YGP-containing serum, mixed standards and YGP extract for $24 \mathrm{hrs,} \mathrm{respectively,} \mathrm{and} \mathrm{assessed} \mathrm{later.} \mathrm{Images} \mathrm{show} \mathrm{immunostaining} \mathrm{of} \mathrm{SH-SY5Y} \mathrm{cells} \mathrm{(I0x}$ magnification). (B) Results of GAP-43 are expressed as the mean \pm SD indicated as percentage of the respective normal cells and analyzed using one-way ANOVA with Least-Significant Difference (LSD) analysis followed by least significant. ${ }^{\Delta} P<0.05,{ }^{\Delta \Delta} P<0.0$ I vs control group cells; ${ }^{\# \#} P<0.0$ I vs model group cells. $n=4$ per group.

a result, both YGP-containing serum and the six components were equally effective, and there were no differences between the groups. It indicated that YGP-containing serum played its pharmacological role through songorine, benzoylhypaconitine, benzoylmesaconitine, neoline, karacoline and sweroside. Furthermore, both the six components and the YGP extract were equally effective, and there were also no differences between the groups. It indicated that the six components might be the main active constituents in YGP for the treatment of MS.

\section{Conclusion}

A rapid, sensitive and accurate LC-MS/MS method was developed and validated for the simultaneous determination of pharmacokinetics in rat plasma. Six bioactive components were detected in rat plasma, including songorine, benzoylhypaconitine, benzoylmesaconitine, neoline, karacoline and sweroside, which were rapidly absorbed after administration. The method validation showed good linearity for all six bioactive components in a wide range with acceptable selectivity, intra- and inter-batch accuracy and precision. Furthermore, the six bioactive components showed good neuroprotective effects with increased expression of CREB and GAP-43, and the results were the same as the YGP extract and the YGP-containing serum in vitro. Therefore, these components were confirmed as the major bioactive components in YGP. The acquired data would be helpful for understanding the pharmacological and medicine material foundation of YGP. However, the components of YGP, the therapeutic 
targets or signaling pathways of these components of YGP are still unclear. In future studies, the molecular mechanisms of them on nerve regeneration will be investigated thoroughly in EAE rat model, and the effect of components will be explored in vivo in future experiments.

\section{Acknowledgments}

This work was supported by the National Natural Science Foundation of China (grant numbers 81873252 and 81573898) and the Beijing Natural Science Foundation (grant numbers 7182020 and 7132031).

\section{Disclosure}

The authors report no conflicts of interest in this work.

\section{References}

1. Ding Q, Ouyang J, Wu S, et al. Influence to Th17/Treg protein expression level in POF mice of you Guiwan. China $J$ of TCM \& Pharm. 2013;28(4):1091-1093.

2. Chinese Pharmacopeia Commission. Pharmacopeia of the People's Republic of China (Version 2015). Beijing: Chinese Pharmacopeia Commission, China Medical Science Press; 2015.

3. Ji X, Liu H, An C, et al. You-Gui pills promote nerve regeneration by regulating netrin 1, DCC and Rho family GTPases RhoA, Racl, Cdc 42 in C57BL/6 mice with experimental autoimmune encephalomyelitis. $J$ Ethnopharmacol. 2016;187:123-133. doi:10.1016/j.jep.2016.04.025

4. Keegan BM, Noseworthy JH. Multiple sclerosis. Annu Rev Med. 2002;53:285-302. doi:10.1146/annurev.med.53.082901.103909

5. Simmons SB, Pierson ER, Lee SY, Goverman JM. Modeling the heterogeneity of multiple sclerosis in animals. Trends Immunol. 2013;34(8):410-422. doi:10.1016/j.it.2013.04.006

6. Mantamadiotis T, Lemberger T, Bleckmann SC, et al. Disruption of CREB function in brain leads to neurodegeneration. Nat Genet 2002;31(1):47-54. doi:10.1038/ng882

7. Lonze BE, Ginty DD. Function and regulation of creb family transcription factors in the nervous system. Neuron. 2002;35(4):605-623.

8. Riccio A, Ahn S, Davenport CM, Blendy JA, Ginty DD. Mediation by a creb family transcription factor of ngf-dependent survival of sympathetic neurons. Science. 1999;286(5448):2358-2361.

9. Bonni A, Brunet A, West AE, Datta SR, Takasu MA, Greenberg ME. Cell survival promoted by the Ras-MAPK signaling pathway by transcription-dependent and -independent mechanisms. Science. 1999;286(5443):1358-1362.

10. Skene J, Jacobson R, Snipes G, McGuire CB, Norden JJ, Freeman JA. A protein induced during nerve growth (GAP-43) is a major component of growth-cone membranes. Science. 1986;233(4765):783-786.

11. Gianola S, Rossi F. Gap-43 overexpression in adult mouse purkinje cells overrides myelin-derived inhibition of neurite growth. Eur $J$ Neurosci. 2004;19(4):819-830.

12. Tikhomirova MS, Karpenko MN, Kirik OV, Sukhorukova EG, Korzhevskiǐ DÉ, Klimenko VM. GAP-43 and its proteolytic fragment in spinal cord cells of rats with experimental autoimmune encephalomilitis. Ross Fiziol Zh Im I M Sechenova. 2015;101(1):74-84.

13. Jacobson RD, Virág I, Skene JH. A protein associated with axon growth, gap-43, is widely distributed and developmentally regulated in rat cns. $J$ Neurosci. 1986;6(6):1843-1855.

14. Teunissen CE, Dijkstra CD, Jasperse B, et al. Growth-associated protein 43 in lesions and cerebrospinal fluid in multiple sclerosis. Neuropathol Appl Neurobiol. 2006;32(3):318-331. doi:10.1111/ j.1365-2990.2006.00730.x
15. Zhang L, Wang P, Ying J, et al. Yougui pills attenuate cartilage degeneration via activation of TGF- $\beta /$ Smad signaling in chondrocyte of osteoarthritic mouse model. Front Pharmacol. 2017;8:611-621. doi:10.3389/fphar.2017.00611

16. Liu H, Qiu F, Bian B, et al. Integrating qualitative and quantitative assessments of Yougui pill, an effective traditional Chinese medicine, by HPLC-LTQ-Orbitrap-MSn and UPLC-QqQ-MS/MS. Anal Methods. 2017;9:3485-3496. doi:10.1039/C7AY00259A

17. Zhang RX, Li MX, Jia ZP. Rehmannia glutinosa: review of botany, chemistry and pharmacology. J Ethnopharmacol. 2008;117(2):199214. doi:10.1016/j.jep.2008.02.018

18. Donnapee S, Li J, Yang X, et al. Cuscuta chinensis Lam.: a systematic review on ethnopharmacology, phytochemistry and pharmacology of an important traditional herbal medicine. J Ethnopharmacol. 2014;157:292-308. doi:10.1016/j.jep.2014.09.032

19. Han J, Tan P, Li Z, et al. Fuzi attenuates diabetic neuropathy in rats and protects schwann cells from apoptosis induced by high glucose. PLoS One. 2014;9(1):e86539. doi:10.1371/journal. pone. 0086539

20. Suzuki T, Miyamoto K, Yokoyama N, et al. Processed aconite root and its active ingredient neoline may alleviate oxaliplatin-induced peripheral neuropathic pain. J Ethnopharmacol. 2016;186:44-52. doi:10.1016/j.jep.2016.03.056

21. Zhou H, Zhang P, Hou Z, et al. Research on the relationships between endogenous biomarkers and exogenous toxic substances of acute toxicity in Radix Aconiti. Molecules. 2016;21:1623. doi:10.3390/ molecules21121623

22. Zhang H, Wu Q, Li W, et al. Absorption and metabolism of three monoester-diterpenoid alkaloids in Aconitum carmichaeli after oral administration to rats by HPLC-MS. J Ethnopharmacol. 2014;154 (3):645-652. doi:10.1016/j.jep.2014.04.039

23. Wang J, van der Heijden R, Spijksma G, et al. Alkaloid profiling of the Chinese herbal medicine Fuzi by combination of matrix-assisted laser desorption ionization mass spectrometry with liquid chromatography-mass spectrometry. J Chromatogr A. 2009;1216(11):21692178. doi:10.1016/j.chroma.2008.11.077

24. Khan H, Nabavi SM, Sureda A, et al. Therapeutic potential of songorine, a diterpenoid alkaloid of the genus Aconitum. Eur $J$ Med Chem. 2017;153:29-33. doi:10.1016/j.ejmech.2017.10.065

25. He YM, Zhu S, Ge YW, et al. The anti-inflammatory secoiridoid glycosides from gentianae scabrae radix: the root and rhizome of Gentiana scabra. J Nat Med. 2015;69(3):303-312. doi:10.1007/s11418-015-0894-8

26. Kou S, Zheng Q, Wang Y, et al. Zuo-Gui and You-Gui pills, two traditional Chinese herbal formulas, downregulated the expression of NogoA, NgR, and RhoA in rats with experimental autoimmune encephalomyelitis. $J$ Ethnopharmacol. 2014;158(Pt A):102-112. doi:10.1016/j.jep.2014.10.007

27. Zappia E, Casazza S, Pedemonte E, et al. Mesenchymal stem cells ameliorate experimental autoimmune encephalomyelitis inducing Tcell anergy. Blood. 2005;106(5):1755-1761. doi:10.1182/blood-200504-1496

28. Zheng Q, Yang T, Fang L, et al. Effects of Bu Shen Yi Sui capsule on Th17/Treg cytokines in C57BL/6 mice with experimental autoimmune encephalomyelitis. BMC Complement Altern Med. 2015;15:60. doi:10.1186/s12906-015-0572-0

29. Huo LR, Liang JT, Zou JH, Wang L-Y, Li Q, Wang X-M. Possible novel roles of poly(rC)-binding protein 1 in SH-SY5Y neurocytes: an analysis using a dynamic bayesian network. Neurosci Bull. 2012;28 (3):282-290. doi:10.1007/s12264-012-1242-6

30. Yang X, Yao W, Shi H, et al. Paeoniflorin protects schwann cells against high glucose induced oxidative injury by activating $\mathrm{Nrf} 2$ / ARE pathway and inhibiting apoptosis. $J$ Ethnopharmacol. 2016;185:361-369. doi:10.1016/j.jep.2016.03.031

31. US Food and Drug Administration. Guidance for Industry, Bioequivalence: Blood Level Bioequivalence Study. Silver Spring, MD: FDA; 2014. 
32. European Medicines Agency. Guideline on Bioanalytical Method Validation, EMEA/CHMP/EWP/192217/2009. Amsterdam: Committee for Medicinal Products for Human Use, European Medicines Agency; 2011.

33. Qiu F, Fu S, Zhou S, Yang S, Yang M. Quantification and pharmacokinetics of Taiwanin e methyl ether in rats by liquid chromatography-tandem mass spectrometry. Biomed Chromatogr. 2013;27 (2):233-239. doi:10.1002/bmc.2781

34. Qiu F, Fu S, Zhang X, Gong M, Wang M. Application of a sensitive and specific LC-MS/MS method for determination of eriodictyol-8-c$\beta$-d-glucopyranoside in rat plasma for a bioavailability study. Biomed Chromatogr. 2015;29(2):220-225. doi:10.1002/bmc.3263

35. Qiu F, Gu Y, Wang T, et al. Quantification and pharmacokinetics of crizotinib in rats by liquid chromatography-tandem mass spectrometry. Biomed Chromatogr. 2016;30(6):962-968. doi:10.1002/bmc.3636

36. Qiu F, Zhao X, Lu X, Wang M, Gong M. Hplc-esi-ms/ms validation and pharmacokinetics of kalopanaxsaponin a in rats. RSC $A d v$. 2015;5(10):7260-7266. doi:10.1039/C4RA14264K
37. Liu H, Qiu F, Zhao H, Bian B, Wang L. Simultaneous high-performance liquid chromatography-tandem mass spectrometric quantification of six bioactive components in rat plasma after oral administration of Yougui pill. J Sep Sci. 2019;4:1-11.

38. Wang Y, Kou S, Gu L, et al. Effects of Zuogui Pill, and Yougui Pill, on the expression of brain-derived neurotrophic factor and cyclic adenosine monophosphate/protein kinase A signaling transduction pathways of axonal regeneration in model rats with experimental autoimmune encephalomyelitis. Chin J Integr Med. 2012;20(1):2430. doi:10.1007/s11655-012-1236-2

39. Wang L, Zhao H, Fan Y, et al. Research on the mechanism of Zuogui Pill and Yougui Pill in promoting axonal regeneration in model rats of autoimmune encephalomyelitis. Chin J Integr Med. 2010;16 (2):167-172. doi:10.1007/s11655-010-0167-z

\section{Publish your work in this journal}

Drug Design, Development and Therapy is an international, peerreviewed open-access journal that spans the spectrum of drug design and development through to clinical applications. Clinical outcomes, patient safety, and programs for the development and effective, safe, and sustained use of medicines are a feature of the journal, which has also been accepted for indexing on PubMed Central. The manuscript management system is completely online and includes a very quick and fair peer-review system, which is all easy to use. Visit http://www. dovepress.com/testimonials.php to read real quotes from published authors. 